\title{
Edge Waves in Plates with Fixed Faces and Various Boundary Conditions on the Front Edge
}

\section{R. V. Ardazishvili, M. V. Wilde, L. Yu. Kossovich}

Saratov State University, 83, Astrakhanskaya st., 410012, Saratov, Russia, ardazishvili.roman@yandex.ru, mv_wilde@mail.ru, president@sgu.ru

This paper is concerned with the propagation of surface waves in plates subject to free or mixed boundary conditions on the front edge. Symmetric and antisymmetric waves in plates with fixed faces are considered. Asymptotic analysis is performed, which shows that there is an infinite spectrum of higher order edge waves in plates. Asymptotics of phase velocity are obtained for large values of wave number. It is demonstrated that in the short-wave limit the phase velocity of all higher order edge waves tends to the velocity of Rayleigh wave or shear wave, depending on the boundary conditions on the front edge.

Key words: surface waves, edge waves, Rayleigh wave, shear wave, asymptotic methods.

\section{References}

1. Belubekian M. V. Surface waves in elastic medium. Problems in Solid Mechanics. Institute of mechanics, National Academy of Sciences of the Republic of Armenia, Yerevan, 1997, pp. 79-96 (in Russian).

2. Kaplunov J. D., Prikazchikov D. A., Rogerson G. A. On three dimesoinal edge waves in prestressed incompressible elastic solids. J. Acoust. Soc. Am., 2005, vol. 118, no. 5, pp. 2975-2983. DOI: $10.1121 / 1.2062487$.

3. Zernov V., Kaplunov J. D. Three-dimensional edge waves in plates. Proc. R. Soc. Lond. A., 2008, vol. 464, pp. 301-318.

4. Wilde M.V., Kaplunov J. D., Kossovich L. Yu. Edge

and interface resonance phenomena in elastic bodies. Moscow, FIZMATLIT, 2010. 280 p. (in Russian).

5. Ardazishvili R. V., Wilde M. V., Kossovich L. Yu. Antisymmetric Higher Order Edge Waves in Plates. Izv. Saratov Univ. (N.S.), Ser. Math. Mech. Inform., 2013, vol. 13, iss. 1, pp. 50-56 (in Russian).

6. Ardazishvili R. V. Three-dimensional surface wave for mixed boundary conditions on the surface. Proceedings of Young Scientists School-Conference MECHANICS-2013, Tsakhkadzor, Armenia, 2013, pp. 74-79.

УДК 681.03.06:531.383:532.516

\section{НЕЛИНЕЙНЫЕ ВОЛНЫ ДЕФОРМАЦИЙ В ГЕОМЕТРИЧЕСКИ И ФИЗИЧЕСКИ НЕЛИНЕЙНОЙ ВЯЗКОУПРУГОЙ ЦИЛИНДРИЧЕСКОЙ ОБОЛОЧКЕ, СОДЕРЖАЩЕЙ ВЯЗКУЮ НЕСЖИМАЕМУЮ ЖИДКОСТЬ И ОКРУЖЕННОЙ УПРУГОЙ СРЕДОЙ}

\author{
А. Ю. Блинкова ${ }^{1}$, Ю. А. Блинков ${ }^{2}$, С. В. Иванов ${ }^{3}$, Л. И. Могилевич ${ }^{4}$
}

\begin{abstract}
${ }^{1}$ Ассистент касредры теплогазоснабжение, вентиляция, водообеспечение и прикладная гидрогазодинамика, Саратовский государственный технический университет им. Гагарина Ю. А., anblinkova@yandex.ru

${ }^{2}$ Доктор фризико-математических наук, заведующий кафедрой математического и компьютерного моделирования, Саратовский государственный университет им. Н. Г. Чернышевского, BlinkovUA@info.sgu.ru

${ }^{3}$ Кандидат фризико-математических наук, старший преподаватель кафредры математического и компьютерного моделирования, Саратовский государственный университет им. Н. Г. Чернышевского, evilgraywolf@gmail.com

${ }^{4}$ Доктор технических наук, просрессор кафредры высшей и прикладной математики, Московский государственный университет путей сообщения (Поволжский фрилиал), Саратов, Mogilevich@sgu.ru

Настоящее исследование посвящено анализу распространения нелинейных продольных волн десрормаций в цилиндрической оболочке, окруженной упругой средой и содержащей вязкую несжимаемую жидкость внутри. Физические свойства оболочки определяются уравнениями квадратичной теории вязкоупругости, учитывающей линейную упругость объемных десрормаций. Проблемы распространения волн в вязкоупругих и нелинейных тонкостенных конструкциях, в том числе цилиндрических оболочках без взаимодействия с вязкой несжимаемой жидкостью, рассмотрены ранее с позиции теории солитонов. Наличие жидкости потребовало разработки новой математической модели и компьютерного моделирования процессов, происходящих в рассматриваемой системе.
\end{abstract}

Ключевые слова: нелинейные волны, вязкая несжимаемая жидкость, вязкоупругие цилиндрические оболочки, окружающая упругая среда. 


\section{1. ПОСТАНОВКА ЗАДАЧИ}

Волновые процессы в вязкоупругих и нелинейных вязкоупругих оболочках, не взаимодействующих с вязкой жидкостью, рассмотрены в [1-3].

Получим уравнения динамики с учётом наличия вязкой несжимаемой жидкости в цилиндрической оболочке с помощью асимптотических методов для решения связанной задачи гидроупругости с соответствующими граничными условиями. Рассмотрим бесконечно длинную вязкоупругую цилиндрическую оболочку, окруженную упругой средой, внутри которой находится вязкая несжимаемая жидкость.

Уравнения движения вязкой несжимаемой жидкости и уравнение неразрывности в цилиндрической системе координат $r, \vartheta, x$ записываются в случае осесимметричного течения в виде [4]

$$
\frac{\partial \bar{V}}{\partial t}+\operatorname{grad} \frac{1}{2} V^{2}+\operatorname{rot} \bar{V} \times \bar{V}+\frac{1}{\rho} \operatorname{grad} \cdot p=-\nu \operatorname{rot} \operatorname{rot} \bar{V}, \quad \operatorname{div} \bar{V}=0,
$$

где $\bar{V}-$ вектор скорости жидкости, $t-$ время, $\rho-$ плотность, $p-$ давление, $\nu-$ кинематический коэффициент вязкости.

На границах с оболочками выполняются условия прилипания жидкости

$$
\frac{\partial U}{\partial t}=V_{x}+U \frac{\partial V_{x}}{\partial x}-W \frac{\partial V_{x}}{\partial r}, \quad-\frac{\partial W}{\partial t}=V_{r}+U \frac{\partial V_{r}}{\partial x}-W \frac{\partial V_{r}}{\partial r} .
$$

Здесь $U$ - продольное упругое перемещение оболочек по оси $x, V_{x}, V_{r}$ - проекции вектора скорости жидкости $\bar{V}$ на оси цилиндрической системы координат, $W$ - прогиб, положительный к центру кривизны оболочки.

В случае осевой симметрии, используя гипотезу Кирхгофа - Лява, имеем связь между компонентами деформаций $\varepsilon_{x}, \varepsilon_{y}$ перемещений [5]

$$
\varepsilon_{x}=\frac{\partial U}{\partial x}-z \frac{\partial^{2} W}{\partial x^{2}}+\frac{1}{2}\left(\frac{\partial U}{\partial x}-z \frac{\partial^{2} W}{\partial x^{2}}\right)^{2}+\frac{1}{2}\left(\frac{\partial W}{\partial x}\right)^{2}, \quad \varepsilon_{y}=-\frac{1}{R} W
$$

где $R$ - радиус срединой поверхности оболочки, $z$ - расстояние от нее. Связь между компонентами напряжений $\sigma_{x}, \sigma_{y}$ и деформаций зададим уравнениями квадратичной теории вязкоупругости [6], учитывающей линейную упругость объёмных деформаций:

$$
\begin{aligned}
& \sigma_{x}=\frac{E}{1-\mu_{0}^{2}}\left(\varepsilon_{x}+\mu_{0} \varepsilon_{y}\right)-\frac{E}{1+\mu_{0}} \alpha \int_{-\infty}^{t} e^{-\beta(t-\tau)}\left(1+a \varepsilon_{u}^{2}\right) e_{x} d \tau, \\
& \sigma_{y}=\frac{E}{1-\mu_{0}^{2}}\left(\varepsilon_{y}+\mu_{0} \varepsilon_{x}\right)-\frac{E}{1+\mu_{0}} \alpha \int_{-\infty}^{t} e^{-\beta(t-\tau)}\left(1+a \varepsilon_{u}^{2}\right) e_{y} d \tau .
\end{aligned}
$$

Здесь $E$ - модуль Юнга, $\mu_{0}$ - коэффициент Пуассона материала оболочек (считая их одинаковыми), $t-$ время, $a, \beta, \rho-$ параметры вязкоупругости, $\varepsilon_{u}^{2}-$ квадрат интенсивности деформаций, $e_{x}, e_{y}-$ компоненты девиатора деформаций:

$$
\varepsilon_{u}^{2}=\frac{4}{3}\left(\varepsilon_{x}^{2}+\varepsilon_{y}^{2}-\varepsilon_{x} \varepsilon_{y}\right) ; \quad e_{x}=\frac{2}{3} \varepsilon_{x}-\frac{1}{3} \varepsilon_{y}, \quad e_{y}=\frac{2}{3} \varepsilon_{y}-\frac{1}{3} \varepsilon_{x} .
$$

Разлагая функции $\left(1+a \varepsilon_{u}^{2}\right) e_{x},\left(1+a \varepsilon_{u}^{2}\right) e_{y}$ в ряд Тейлора по степеням $(t-\tau)$, при условии $\beta t \gg 1$ сохраняем два члена разложения их формул (4), (5), получим приближенные уравнения состояния [1-3]

$$
\begin{aligned}
& \sigma_{x}=\frac{E}{1-\mu_{0}^{2}}\left(\varepsilon_{x}+\mu_{0} \varepsilon_{y}\right)+\mathrm{p}\left[\frac{2}{3} \varepsilon_{x}-\frac{1}{3} \varepsilon_{y}+a\left(e_{u}^{2} e_{x}\right)\right], \\
& \sigma_{y}=\frac{E}{1-\mu_{0}^{2}}\left(\varepsilon_{y}+\mu_{0} \varepsilon_{x}\right)+\mathrm{p}\left[\frac{2}{3} \varepsilon_{y}-\frac{1}{3} \varepsilon_{x}+a\left(\varepsilon_{u}^{2} e_{y}\right)\right],
\end{aligned}
$$


где введен оператор р такой, что

$$
\mathrm{p} f=\frac{E}{1+\mu_{0}}\left(\frac{\alpha}{\beta^{2}} \frac{\partial f}{\partial t}-\frac{\alpha}{\beta} f\right) .
$$

Вычисляя с использованием (6) усилия и моменты по формулам [5]

$$
N_{x}=\int_{-h_{0} / 2}^{h_{0} / 2} \sigma_{x} \mathrm{~d} z, \quad N_{y}=\int_{-h_{0} / 2}^{h_{0} / 2} \sigma_{y} \mathrm{~d} z, \quad M_{x}=\int_{-h_{0} / 2}^{h_{0} / 2} \sigma_{x} z \mathrm{~d} z, \quad M_{y}=\int_{-h_{0} / 2}^{h_{0} / 2} \sigma_{y} z \mathrm{~d} z,
$$

и подставим (9) в систему уравнений динамических оболочек [5]:

$$
\begin{gathered}
\frac{\partial N_{x}}{\partial x}-\rho_{0} h_{0} \frac{\partial^{2} U}{\partial t^{2}}=-q_{x} \\
\frac{\partial^{2} M_{x}}{\partial x^{2}}+\frac{1}{R} N_{y}+\frac{\partial}{\partial x}\left(\frac{\partial W}{\partial x} N_{x}\right)-\rho_{0} h_{0} \frac{\partial^{2} W}{\partial t^{2}}= \\
=-q_{n}-\rho_{0} h_{0}\left[k_{1} \frac{c_{0}^{2}}{l^{2}} W-k_{2} \frac{c_{0}^{2} l^{2}}{R^{6}} W^{3}-2 t_{1} c_{0}^{2} \frac{\partial^{2} W}{\partial x^{2}}+m \frac{\partial^{2} W}{\partial t^{2}}\right] .
\end{gathered}
$$

Здесь $h_{0}$ - толщина оболочки, $\rho_{0}$ - плотность материала оболочки, $q_{x}, q_{n}-$ напряжения, действующие со стороны жидкости на поверхность оболочки, снесенные на невозмущаемую поверхность оболочки $(W \ll R)$

$$
q_{x}=\left[\rho \nu\left(\frac{\partial V_{x}}{\partial r}+\frac{\partial V_{r}}{\partial x}\right)\right]_{r=R}, \quad q_{n}=\left[-\rho+2 p \nu \frac{\partial V_{r}}{\partial r}\right]_{r=R} .
$$

Выражение в квадратных скобках характеризует реакцию упругой среды, в которой расположена труба кругового сечения Власова-Леонтьева [7]. $k_{1} \frac{c_{0}^{2}}{l^{2}} W-k_{2} \frac{c_{0}^{2} l^{2}}{R^{6}} W^{3}-$ реакция на сдавливание (сжатие), $-2 t_{1} c_{0}^{2} \frac{\partial^{2} W}{\partial x^{2}}-$ реакция на сдвиг, $m \frac{\partial^{2} W}{\partial t^{2}}-$ инерционная реакция. Коэффициенты $k_{1}, k_{2}$, $t_{1}, m$ - определены в [7].

\section{2. ВЫВОД УРАВНЕНИЙ ДИНАМИКИ ОБОЛОЧЕК}

Принимая за характерную длину длину волны деформации $l$, перейдем к безразмерным переменным для исследования уравнений динамики оболочек (3)-(10)

$$
W=w_{m} u_{3}, \quad U=u_{m} u_{1}, \quad t^{*}=\frac{c_{0}}{l} t, \quad x^{*}=\frac{x}{l}, \quad c_{0}=\sqrt{\frac{E}{\rho_{0}\left(1-\mu_{0}^{2}\right)}} .
$$

Здесь $c_{0}-$ скорость звука в материале оболочки.

Положим

$$
\begin{gathered}
\frac{u_{m}}{l}=\varepsilon=o(1), \quad \frac{w_{m}}{R}=O(\varepsilon), \quad a=O\left(\varepsilon^{-1}\right), \quad \frac{\alpha}{\beta}=O(1), \\
\frac{\alpha}{\beta^{2}} \frac{c_{0}}{l}=O(\varepsilon), \quad \frac{R}{l}=O\left(\varepsilon^{1 / 2}\right), \quad \frac{h_{0}}{R}=O(\varepsilon),
\end{gathered}
$$

где $\varepsilon \ll 1-$ малый параметр задачи.

Применим метод двухмасштабных разложений, вводя независимые переменные в виде

$$
\xi=x^{*}-c t^{*}, \quad \tau=\varepsilon t^{*},
$$

где $c$ - безразмерная неизвестная скорость волны, а зависимые переменные представлены в виде разложения по малому параметру $\varepsilon$ :

$$
u_{1}=u_{10}+\varepsilon u_{11}+\ldots, \quad u_{3}=u_{30}+\varepsilon u_{31}+\ldots .
$$


Подставляя (13), (15), (16) в уравнения (11), (12), с учетом оценок (14) получим в нулевом приближении по $\varepsilon$ линейную систему уравнений, из которой следует связь

$$
\frac{w_{m}}{R} u_{30}=\mu_{1} \frac{u_{m}}{l} \frac{\partial u_{10}}{\partial \xi}, \quad \mu_{1}=\frac{\mu_{0}+\frac{1}{3}\left(1-\mu_{0}\right) \frac{\alpha}{\beta}}{1-\frac{2}{3}\left(1-\mu_{0}\right) \frac{\alpha}{\beta}}
$$

и определяется безразмерная скорость волны:

$$
c^{2}=\left[1-\frac{2}{3}\left(1-\mu_{0}\right) \frac{\alpha}{\beta}\right]\left(1-\mu_{1}^{2}\right) .
$$

Из следующего приближения по $\varepsilon$, учитывая (17) и (18), находится уравнение для определения $u_{10}$ :

$$
\begin{gathered}
\frac{\partial^{2} u_{10}}{\partial \xi \partial \tau}+\frac{c}{2} \frac{R^{2}}{l^{2} \varepsilon} \mu_{1}^{2}\left(1+m-\frac{2 t_{1}}{c^{2}}\right) \frac{\partial^{4} u_{10}}{\partial \xi^{4}}+\frac{c}{2} \frac{u_{m}}{l \varepsilon} \frac{\partial u_{10}}{\partial \xi} \frac{\partial^{2} u_{10}}{\partial \xi^{2}}- \\
-\left\{\frac{2}{3} \frac{\left(1-\mu_{0}\right) \frac{\alpha}{\beta}}{c}\left[1+\mu_{1}^{4}+\left(1+\mu_{1}\right)^{4}\right] \frac{a}{\varepsilon}\left(\frac{u_{m}}{l}\right)^{2}+\frac{3}{2 c} \frac{1}{\varepsilon} \frac{u_{m}^{2}}{R^{2}} k_{2} \mu_{1}^{4}\right\}\left(\frac{\partial u_{10}}{\partial \xi}\right)^{2} \frac{\partial^{2} u_{10}}{\partial \xi^{2}}- \\
-\frac{1}{3}\left(1-\mu_{0}\right) \frac{\alpha}{\beta^{2}} \frac{c_{0}}{l \varepsilon}\left(1+\mu_{1}+\mu_{1}^{2}\right) \frac{\partial^{3} u_{10}}{\partial \xi^{3}}+\frac{1}{\varepsilon} \frac{R^{2}}{l^{2}} \frac{\mu_{1}^{2}}{2 c} k_{1} \frac{\partial^{2} u_{10}}{\partial \xi^{2}}=-\frac{l^{2}}{\varepsilon u_{m} \rho_{0} h_{0} c_{0}^{2} 2 c}\left[q_{x}-\mu_{1} \frac{R}{e} \frac{\partial q_{n}}{\partial \xi}\right] .
\end{gathered}
$$

\section{3. ОПРЕДЕЛЕНИЕ НАПРЯЖЕНИЙ ДЕЙСТВУЮЩИХ СО СТОРОНЫ ЖИДКОСТИ}

Для определения правой части уравнения (19) введем безразмерные переменные и параметры

$$
\begin{array}{ll}
V_{r}=w_{m} \frac{c_{0}}{l} v_{r}, & V_{x}=w_{m} \frac{c_{0}}{R_{1}} v_{x}, \quad r^{*}=\frac{r}{R_{1}}, \quad t^{*}=\frac{c_{0}}{l} t, \quad x^{*}=\frac{x}{l}, \\
p=\frac{\rho \nu c_{0} l w_{m}}{R_{1}^{3}} P, & \psi=\frac{R_{1}}{l}=o\left(\varepsilon^{1 / 2}\right), \quad \lambda=\frac{w_{m}}{R_{1}}, \quad \psi \ll 1, \quad \lambda \ll 1 .
\end{array}
$$

Здесь $R_{1}-$ внутренний радиус оболочки.

Подставляя (20) в уравнения гидродинамики (1) и граничные условия (2), представим безразмерные скорость и давление в виде разложения по малому параметру $\lambda$ :

$$
v_{x}=v_{x}^{0}+\lambda v_{x}^{1}+\ldots, \quad v_{r}=v_{r}^{0}+\lambda v_{r}^{1}+\ldots, \quad P=P^{0}+\lambda P^{1}+\ldots .
$$

В нулевом приближении по $\psi\left(\psi \approx 0-\right.$ гидравлическая теория смазки), считая $(\psi)\left(R_{1} c_{0} / \nu\right) \ll 1$ (ползущие течения $[8,9]$ ), и в нулевом приближении по $\lambda$ получаем уравнения гидродинамики (классические уравнения гидродинамической теории смазки):

$$
\frac{\partial P^{0}}{\partial r^{*}}=0, \quad \frac{\partial P^{0}}{\partial x}=\frac{1}{r^{*}} \frac{\partial}{\partial r^{*}}\left(r^{*} \frac{\partial v_{x}^{0}}{\partial r^{*}}\right), \quad \frac{1}{r^{*}} \frac{\partial}{\partial r^{*}}\left(r^{*} v_{r}^{0}\right)+\frac{\partial v_{x}^{0}}{\partial x^{*}}=0,
$$

и граничные условия

$$
\begin{gathered}
r^{*} \frac{\partial v_{x}^{0}}{\partial r^{*}}=0, \quad r^{*} \frac{\partial v_{r}^{0}}{\partial r^{*}}=0 \quad \text { при } \quad r^{*}=0, \\
v_{r}^{0}=-\frac{\partial u_{30}}{\partial t^{*}}, \quad v_{x}^{0}=\frac{u_{m} R_{1}}{W_{m} l} \frac{\partial u_{10}}{\partial t^{*}} \quad \text { при } \quad r^{*}=1 .
\end{gathered}
$$

Из решения задачи (22), (23) следует, что

$$
P^{0}=16 \int\left[\frac{1}{2} \frac{u_{m} R_{1}}{w_{m} l} \frac{\partial u_{10}}{\partial t^{*}}-\int \frac{\partial u_{30}}{\partial t^{*}} \mathrm{~d} x^{*}\right] \mathrm{d} x^{*}, \quad \frac{\partial v_{x}^{0}}{\partial r^{*}}=\frac{1}{2} r^{*} \frac{\partial P_{0}}{\partial x^{*}} .
$$

С принятой точностью по $\varepsilon, \psi, \lambda$ из (6) найдем

$$
q_{x}=\left.\rho \nu \frac{w_{m} c_{0}}{R_{1}^{2}} \frac{\partial v_{x}^{0}}{\partial R^{*}}\right|_{r^{*}=1}, \quad q_{n}=-\rho \nu \frac{w_{m} c_{0}}{R_{1}^{2}} \frac{l}{R_{1}} P^{0},
$$

и выражение в квадратных скобках правой части (19) имеем вид

$$
\left[q_{x}-\mu_{1} \frac{R}{l} \frac{\partial q_{n}}{\partial \varepsilon}\right]=\rho \nu \frac{w_{m} c_{0}}{R_{1}^{2}}\left[\left.\frac{\partial v_{x}^{0}}{\partial r^{*}}\right|_{r^{*}=1}+\mu_{1} \frac{R}{l} \frac{l}{R_{1}} \frac{\partial P^{0}}{\partial x^{*}}\right]=\rho \nu \frac{w_{m} c_{0}}{R_{1}^{2}} \frac{1}{2} \frac{\partial P^{0}}{\partial \xi}\left[1+2 \mu_{1} \frac{R}{R_{1}}\right] .
$$


Учитывая, что были введены переменные (20), (21), и имея соотношения (24), (25), получим:

$$
P^{0}=8 c \frac{u_{m} R_{1}}{w_{m} l}\left[2 \mu_{1} \frac{R}{R_{1}}-1\right] u_{10} .
$$

Следовательно, в правой части уравнения (19) остается выражение

$$
2 \frac{\rho l \nu}{\rho_{0} h_{0} R_{1} c_{0} \varepsilon}\left[1-\left(2 \mu_{1} \frac{R}{R_{1}}\right)^{2}\right] \frac{\partial u_{10}}{\partial \xi}
$$

с принятой точностью по $\psi, \varepsilon$ положим $R_{1} \approx R$. Подставляя (27) в уравнение (19), окончательно получим:

$$
\begin{gathered}
\frac{\partial^{2} u_{10}}{\partial \xi \partial \tau}+\frac{c}{2} \frac{R^{2}}{l^{2} \varepsilon} \mu_{1}^{2}\left(1+m-\frac{2 t_{1}}{c^{2}}\right) \frac{\partial^{4} u_{10}}{\partial \xi^{4}}+\frac{c}{2} \frac{u_{m}}{l \varepsilon} \frac{\partial u_{10}}{\partial \xi} \frac{\partial^{2} u_{10}}{\partial \xi^{2}}- \\
-\left\{\frac{2}{3} \frac{\left(1-\mu_{0}\right) \frac{\alpha}{\beta}}{c}\left[1+\mu_{1}^{4}+\left(1+\mu_{1}\right)^{4}\right] \frac{a}{\varepsilon}\left(\frac{u_{m}}{l}\right)^{2}+\frac{3}{2 c} \frac{1}{\varepsilon} \frac{u_{m}}{R^{2}} k_{2} \mu_{1}^{4}\right\}\left(\frac{\partial u_{10}}{\partial \xi}\right)^{2} \frac{\partial^{2} u_{10}}{\partial \xi^{2}}- \\
-\frac{1}{3}\left(1-\mu_{0}\right) \frac{\alpha}{\beta^{2}} \frac{c_{0}}{l \varepsilon}\left(1+\mu_{1}+\mu_{1}^{2}\right) \frac{\partial^{3} u_{10}}{\partial \xi^{3}}+\frac{1}{\varepsilon} \frac{R^{2}}{l^{2}} \frac{\mu_{1}^{2}}{2 c} k_{1} \frac{\partial^{2} u_{10}}{\partial \xi^{2}}-2\left[1-\left(2 \mu_{1}\right)^{2}\right] \frac{\rho l \nu}{\rho_{0} h_{0} R_{1} c_{0} \varepsilon} \frac{\partial u_{10}}{\partial \xi}=0 .
\end{gathered}
$$

При отсутствии жидкости $(\rho=0)$ последнее слагаемое выпадает, и уравнение (28), превращающееся в уравнение Гарднера - Бюргерса для $\frac{\partial u_{10}}{\partial \xi}$, имеет точное частное решение. В зависимости от физичеких параметров величина $\mu_{1}$ может быть больше $1 / 2$, меньше $1 / 2$ или равна $1 / 2$. Последний случай эквивалентен отсутствию жидкости, но означает, что она не влияет на волну деформации.

Легко видеть, что замена

$$
\frac{\partial u_{10}}{\partial \xi}=c_{3} \varphi, \quad \eta=c_{1} \xi, \quad t=c_{2} \tau
$$

позволяет записать уравнение (28) в виде

$$
\frac{\partial \varphi}{\partial t}+6 \sigma_{0} \varphi \frac{\partial \varphi}{\partial \eta}+\frac{\partial^{3} \varphi}{\partial \eta^{3}}-6 \sigma_{1} \varphi^{2} \frac{\partial \varphi}{\partial \eta}-\sigma_{2} \frac{\partial^{2} \varphi}{\partial \eta^{2}}+\sigma_{3} \frac{\partial \varphi}{\partial \eta}-\sigma \varphi=0 .
$$

Здесь $\sigma=1$ при $\mu_{1}<1 / 2, \sigma=-1$ при $\mu_{1}>1 / 2$ и $\sigma=0$ при $\mu_{1}=1 / 2$.

Постоянные $c_{3}, c_{1}, c_{2}$ определяются формулами

$$
c_{2}=2 \sigma\left[1-\left(2 \mu_{1}\right)^{2}\right] \frac{\rho l \nu}{\rho_{0} h_{0} R_{1} c_{0} \varepsilon}, \quad c_{1}=\left[c_{2} \varepsilon\left(\frac{l}{R}\right)^{2} \frac{2}{c \mu_{1}^{2}\left(1+m-\frac{2 t_{1}}{c^{2}}\right)}\right]^{1 / 3}, \quad c_{3}=6 \frac{c_{2}}{c_{1}} \frac{2 l \varepsilon}{c u_{m}} \sigma_{0} .
$$

При этом вводятся обозначения

$$
\begin{gathered}
6 \sigma_{1}=\frac{c_{3}^{2} c_{1}}{c_{2}}\left[\frac{2}{3} \frac{a}{\varepsilon}\left(\frac{u_{m}}{l}\right)^{2}\left(1-\mu_{0}\right) \frac{\alpha}{\beta} \frac{1+\mu_{1}^{4}+\left(1+\mu_{1}\right)^{4}}{c}+3 k_{2} \frac{1}{\varepsilon} \frac{u_{m}}{R^{2}} \frac{\mu_{1}^{4}}{2 c}\right], \\
\sigma_{2}=\frac{c_{1}^{2}}{c_{2}} \frac{1}{3} \frac{\alpha}{\beta^{2}} \frac{c_{0}}{l \varepsilon}\left(1-\mu_{0}\right)\left(1+\mu_{1}+\mu_{1}^{2}\right), \quad \sigma_{3}=\frac{c_{1}}{c_{2}} \frac{1}{\varepsilon} \frac{R^{2}}{l^{2}} \frac{\mu_{1}^{2}}{2 c} k_{1} .
\end{gathered}
$$

При отсутствии жидкости уравнение (29) превращается в уравнение Гарднера - Бюргерса, имеющее точное частное решение:

$$
\frac{3 \sigma_{0} \mp \sigma_{2} \sqrt{\sigma_{1}} \mp 6 k \sqrt{\sigma_{1}} \operatorname{th}\left\{k \eta+\left(2 k^{3}+\frac{\sigma_{2}^{2} k}{6}-\frac{3}{2} \frac{k \sigma_{0}^{2}}{\sigma_{1}}-\sigma_{3} k\right) t\right\}}{6 \sigma_{1}},
$$

которое можно использовать в качестве начального условия с верхним знаком при $t=0$ для численного решении уравнения (29):

$$
\phi(\eta, t=0)=\frac{3 \sigma_{0}-\sigma_{2} \sqrt{\sigma_{1}}-6 k \sqrt{\sigma_{1}} \operatorname{th}\{k \eta\}}{6 \sigma_{1}} .
$$




\section{4. КОМПЬЮТЕРНОЕ МОДЕЛИРОВАНИЕ}

В работах [10-12] развит подход к построению разностных схем, основанный на построении системы разностных уравнений, получаемой из аппроксимации интегральных законов сохранения и интегральных соотношений, связывающих искомые функции и их производные. В результате разностная схема определяется как условие совместности для данной системы. Таким образом, применение техники базисов Грёбнера позволяет сгенерировать разностные схемы, из которых путем эквивалентных преобразований могут быть получены дискретные аналоги законов сохранения исходных дифференциальных уравнений.

Запишем уравнение (29) в интегральной форме:

$$
\oint_{\partial \Omega}\left(-3 \sigma_{0} \varphi^{2}-\varphi_{\eta \eta}+2 \sigma_{1} \varphi^{3}+2 \sigma_{2} \varphi_{\eta}-\sigma_{3} \varphi\right) d t+\sigma \varphi d \eta-\iint_{\Omega} \sigma \varphi d t d \eta=0
$$

для любой области $\Omega$. Для перехода к дискретной формулировке сопоставим $u_{j}^{n}=\varphi\left(t_{n}, \eta_{j}\right)$ и выберем

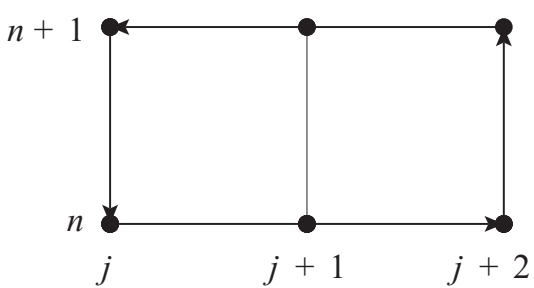

Рис. 1. Базовой контур для уравнения (32) в качестве базового контур, показанный на рис. 1.

Добавим интегральные соотношения

$$
\begin{gathered}
\int_{\substack{\eta_{j} \\
\eta_{j+1}}}^{\eta_{j+1}} u_{\eta} d \eta=u\left(t, \eta_{j+1}\right)-u\left(t, \eta_{j}\right), \\
\int_{\eta_{j}}^{u_{\eta}} u_{\eta \eta} d \eta=u_{\eta}\left(t, \eta_{j+1}\right)-u_{\eta}\left(t, \eta_{j}\right) .
\end{gathered}
$$

Используя для интегрирования по времени и по четным производным по $\eta$ формулу трапеций, а по нечетным производным по $\eta$ формулу среднего значения, и полагая $t_{n+1}-t_{n}=\tau, \eta_{j+1}-\eta_{j}=h$, перепишем соотношения (32), (33) в виде

$$
\begin{aligned}
& \left(-3 \sigma_{0}\left(u^{2^{n}}{ }_{j}+u^{2^{n+1}}-u^{2^{n}}{ }_{j+2}-u^{2^{n+1}}{ }_{j+2}\right)-\left(u_{\eta \eta_{j}}{ }^{n}+u_{\eta \eta_{j}}^{n+1}-u_{\eta \eta_{j+2}}^{n}-u_{\eta \eta_{j+2}}^{n+1}\right)+\right. \\
& +2 \sigma_{1}\left(u^{3}{ }_{j}^{n}+u_{j}^{3}{ }_{j}^{n+1}-u_{j+2}^{3}{ }_{j+2}-u_{j+2}^{3 n+1}\right)+2 \sigma_{2}\left(u_{\eta_{j}}^{n}+u_{\eta_{j}}^{n+1}-u_{\eta_{j+2}}^{n}-u_{\eta_{j+2}}^{n+1}\right)- \\
& \left.-\sigma_{3}\left(u_{j}^{n}+u_{j}^{n+1}-u_{j+2}^{n}-u_{j+2}^{n+1}\right)\right) \cdot \frac{\tau}{2}+\left(u_{j+1}^{n+1}-u_{j+1}^{n}\right) \cdot 2 h-\sigma\left(u_{j+1}^{n+1}+u_{j+1}^{n}\right) \cdot h \tau=0 \text {, } \\
& \left(u_{\eta_{j+1}}^{n}+u_{\eta_{j}}^{n}\right) \cdot \frac{h}{2}=u_{j+1}^{n}-u_{j}^{n}, \quad u_{\eta \eta_{j+1}^{n}}^{n} \cdot 2 h=u_{\eta_{j+2}^{n}}^{n}-u_{\eta_{j}}^{n} .
\end{aligned}
$$

Используя пакет LDA [13] для построения базисов Грёбнера в случае разностных идеалов реализованный в системе компьютерной алгебры Maple, зададим начальные разностные соотношения:

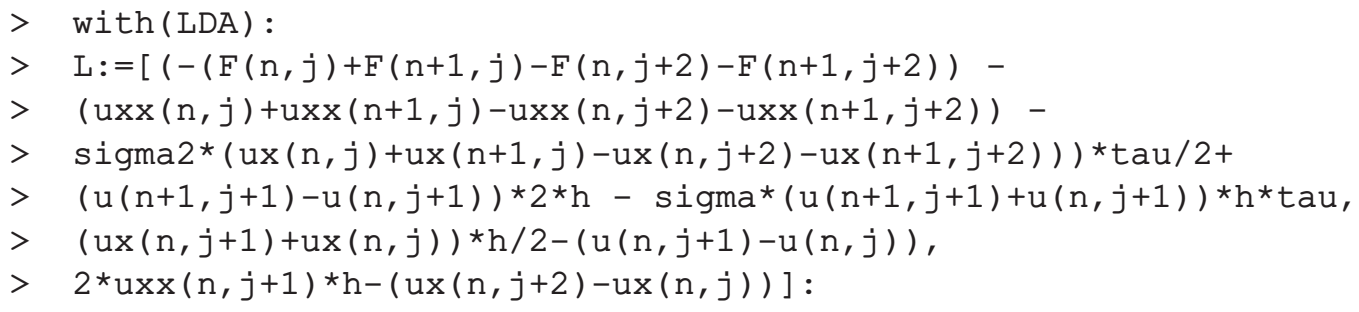

Поскольку пакет [13] работает только в случае линейных разностных идеалов, а исходное дифференциальное уравнение (29) нелинейно, заменим нелинейную часть введением дополнительной функции $F=3 \sigma_{0} \varphi^{2}-2 \sigma_{1} \varphi^{3}+\sigma_{3} \varphi$. За счет выбора допустимого лексикографического упорядочения сначала по функциям $u_{x x} \succ u_{x} \succ u \succ F$, а затем по переменным $n$, $j$, нелинейная часть не будет входить в лидирующие мономы системы при построении базиса Грёбнера:

$>\operatorname{JanetBasis}(L,[n, j],[u x x, u x, u, F], 2)$; 


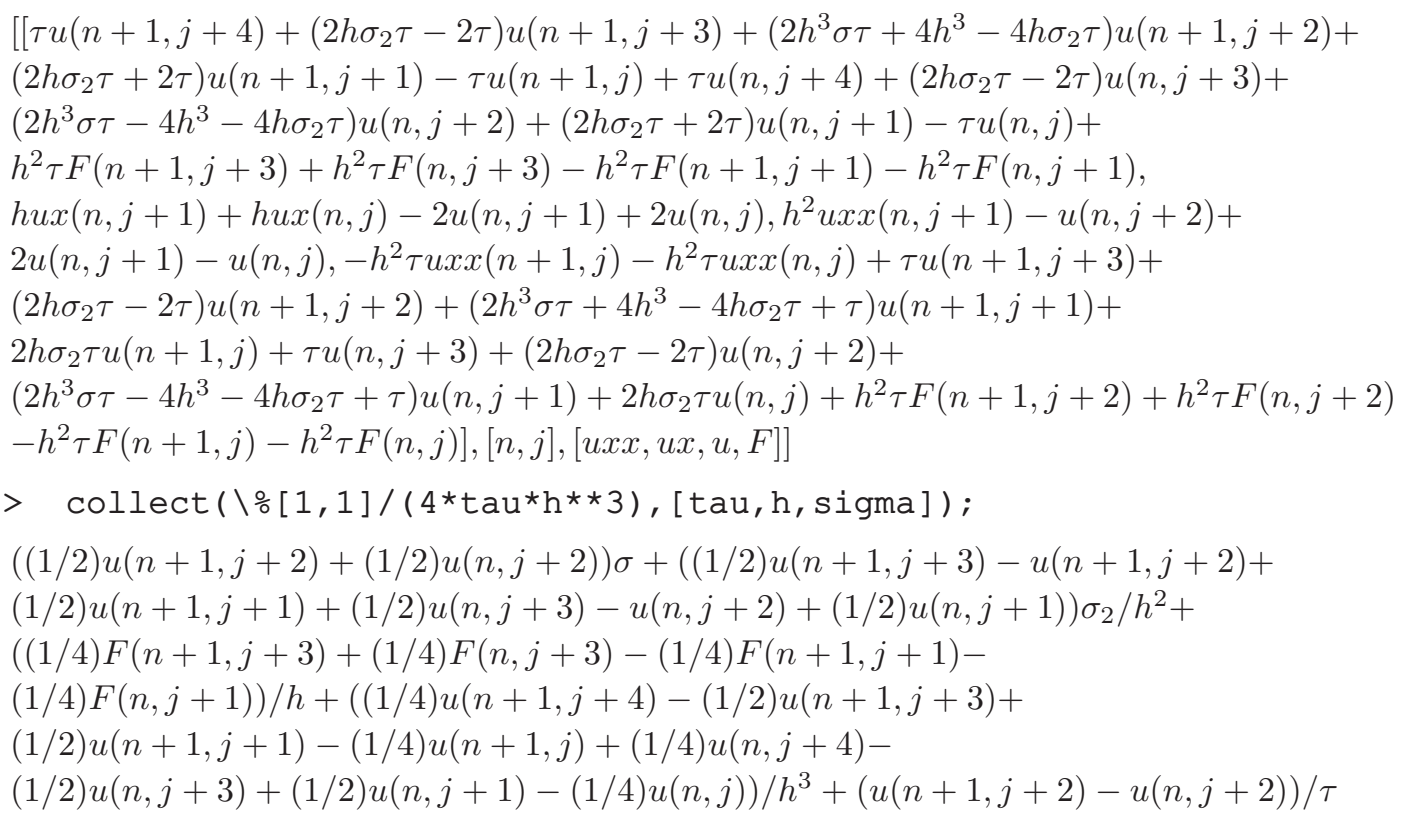

В результате получим следующую разностную схему для уравнения (29), аналогичную схеме Кранка - Николсона для уравнения теплопроводности:

$$
\begin{gathered}
\frac{u_{j}^{n+1}-u_{j}^{n}}{\tau}+3 \sigma_{0} \frac{\left(u_{j+1}^{2}-u_{j-1}^{2}{ }_{j+1}^{n+1}\right)+\left(u_{j+1}^{2^{n}}-u_{j-1}^{2^{n}}\right)}{4 h}+ \\
+\frac{\left(u_{j+2}^{n+1}-2 u_{j+1}^{n+1}+2 u_{j-1}^{n+1}-u_{j-2}^{n+1}\right)+\left(u_{j+2}^{n}-2 u_{j+1}^{n}+2 u_{j-1}^{n}-u_{j-2}^{n}\right)}{4 h^{3}}- \\
-2 \sigma_{1} \frac{\left(u_{j+1}^{3}{ }_{j+1}^{n+1}-u_{j-1}^{3}\right)+\left(u_{j+1}^{3+1}-u_{j-1}^{3}\right)}{4 h}+\sigma_{2} \frac{\left(u_{j+1}^{n+1}-2 u_{j}^{n+1}+u_{j-1}^{n+1}\right)+\left(u_{j+1}^{n}-2 u_{j}^{n}+u_{j-1}^{n}\right)}{2 h^{2}}+ \\
+2 \sigma_{3} \frac{\left(u_{j+1}^{n+1}-u_{j-1}^{n+1}\right)+\left(u_{j+1}^{n}-u_{j-1}^{n}\right)}{4 h}-\sigma \frac{u_{j}^{n+1}+u_{j}^{n}}{2}=0 .
\end{gathered}
$$

Полученные неявные разностные схемы имеют квадратичную и кубическую нелинейность для следующего временного слоя. При построении решения использована следующая линеаризация:

$$
\begin{gathered}
v_{k+1}^{2}=v_{k+1}^{2}-v_{k}^{2}+v_{k}^{2}=\left(v_{k+1}-v_{k}\right)\left(v_{k+1}+v_{k}\right)+v_{k}^{2} \approx v_{k+1} \cdot 2 v_{k}-v_{k}^{2}, \\
v_{k+1}^{3}=v_{k+1}^{3}-v_{k}^{3}+v_{k}^{3}=\left(v_{k+1}-v_{k}\right)\left(v_{k+1}^{2}+v_{k+1} v_{k}+v_{k}^{2}\right)+v_{k}^{3} \approx v_{k+1} \cdot 3 v_{k}^{2}-2 v_{k}^{3} .
\end{gathered}
$$

Количество итераций для достижения точности $10^{-12}$ на следующем временном слое, как правило, не превышало 3. Шаг по времени $t$ брался равным половине шага по переменной $\eta$. Программа расчета была написана на языке Python с использованием пакета SciPy.

Как показано на рис. 2, а и б и как можно увидеть из точного решения (30) уравнения (29) при $\sigma=0$ упругая среда, окружающая оболочку, приводит к увлечению скорости нелинейной волны деформации.

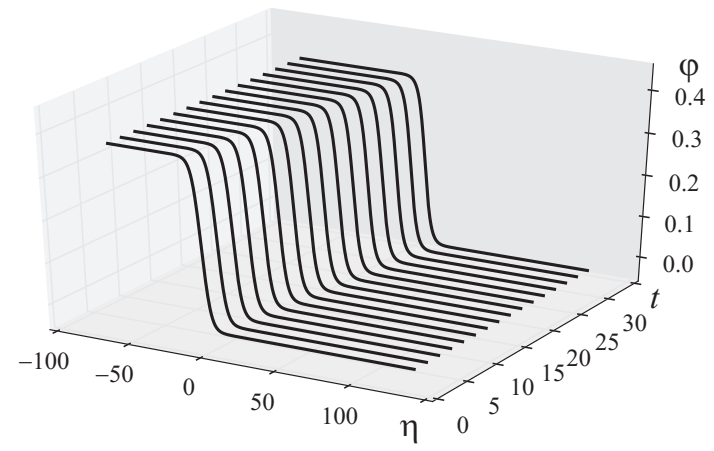

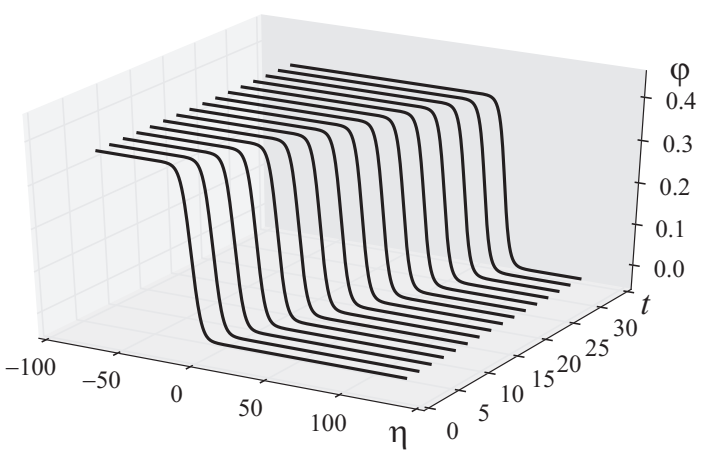

б

Рис. 2. Графики численного решения уравнений (29) при $\sigma_{3}=0(a)$ и $\sigma_{3}=2$ (б) для $\sigma_{0}=1, \sigma_{1}=1, \sigma_{2}=1.8$, $\sigma=0$ с начальным условием (31) для $\varphi$ с $k=0.2$ 
Выполненные вычислительные эксперименты позволили оценить влияние вязкой несжимаемой жидкости на поведение нелинейной волны деформации в бесконечно длинной оболочке в зависимости от введенного параметра $\mu_{1}$, характеризующего вязкоупругие свойства материала оболочки, аналогичного коэффициенту Пуассона для упругой среды. Наблюдается рост амплитуды волны при $\mu_{1}<1 / 2$ на рис. 3, а из-за нарушения баланса между нелинейностью и дисперсией с учетом демпфирования в пользу нелинейности и падения амплитуды волны при $\mu_{1}>1 / 2$ на рис. 3, б из-за нарушения баланса между нелинейностью и дисперсией с учетом демпфирования в пользу дисперсии и диссипации. При $\mu_{1}=1 / 2$ влияние жидкости перестает сказываться.

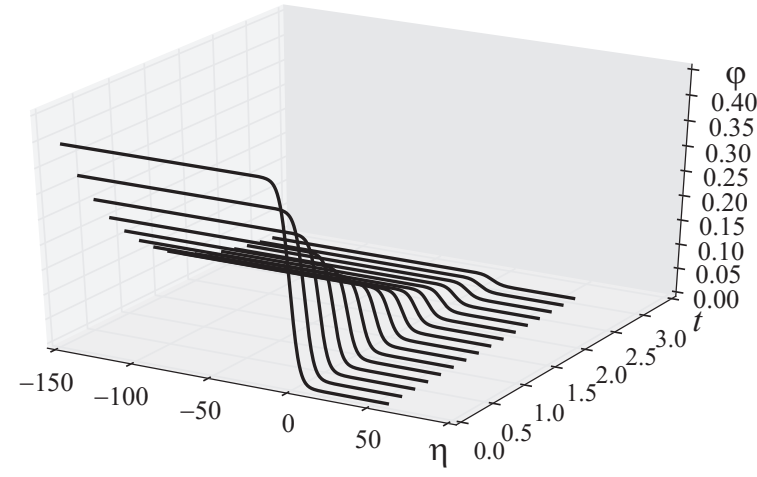

$a$

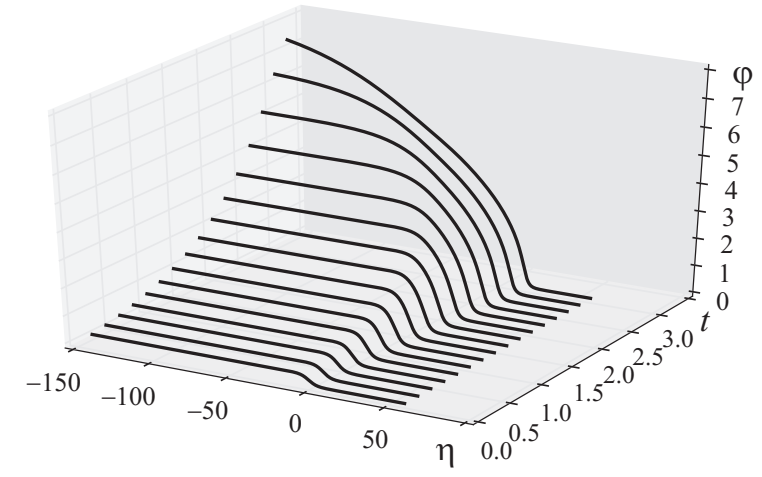

$\sigma$

Рис. 3. Графики численного решения уравнений (29) при $\sigma=-1(a)$ и $\sigma=1$ (б) для $\sigma_{0}=1, \sigma_{1}=1, \sigma_{2}=1.8$, $\sigma_{3}=2$ с начальным условием (31) для $\varphi$ с $k=0.2$

\section{ЗАКЛЮЧЕНИЕ}

Построены новые математические модели в виде нелинейных уравнений в частных производных, обобщающих уравнения Гарднера - Бюргерса, описывающие волновые процессы в вязкоупругих бесконечно длинных цилиндрических оболочках с вязкой несжимаемой жидкостью внутри. На основе применения техники базисов Грёбнера сгенерированы разностные схемы, из которых путем эквивалентных преобразований могут быть получены дискретные аналоги законов сохранения исходных дифференциальных уравнений. Упругая среда, окружающая оболочку, приводит к увеличению скорости нелинейной волны деформации. Выполненные вычислительные эксперименты позволили оценить влияние вязкой несжимаемой жидкости на поведение нелинейной волны деформации в бесконечно длинной оболочке в зависимости от введенного параметра $\mu_{1}$, характеризующего вязкоупругие свойства материала оболочки, аналогичного коэффициенту Пуассона для упругой среды. Наблюдается рост амплитуды волны при $\mu_{1}<1 / 2(\sigma=1)$ и падения амплитуды волны при $\mu_{1}>1 / 2(\sigma=-1)$. При $\mu_{1}=1 / 2(\sigma=0)$ влияние жидкости перестает сказываться. Распространение нелинейных волн деформаций в вязкоупругих бесконечно длинных цилиндрических оболочках, содержащих внутри вязкую несжимаемую жидкость, сопровождается сглаживанием профиля волны и колебаний возникающих за ее фронтом за счет диссипации.

Работа выполнена при финансовой поддержке РФФИ (проект № 13-01-00049-а).

\section{Библиографический список}

1. Землянухин А. И., Могилевич Л. И. Нелинейные волны в цилиндрических оболочках : солитоны, симметрии, эволюция. Саратов : Изд-во Сарат. гос. техн. ун-та, 1999. 132 с.

2. Аршинов Г. А., Землянухин А. И., Могилевич Л. И. Двумерные уединенные волны в нелинейной вязкоупругой деформируемой среде // Акустический журн. 2000. Т. 46, № 1. С. 116-117.

3. Аршинов Г. А., Могилевич Л. И. Статические и динамические задачи вязкоупругости. Саратов : Изд-во Сарат. гос. аграрного ун-та, 2000. 152 с.
4. Лойцянский Л. Г. Механика жидкости и газа. М. : Дрофа, 2003. 840 с.

5. Вольмир А. С. Нелинейная динамика пластинок и оболочек. М. : Наука, 1972. 328 с.

6. Москвитин В. В. Сопротивление вязко-упругих материалов. М. : Наука, 1972. 328 с.

7. Власов В. З., Леонтьев Н. Н. Балки, плиты и оболочки на упругом основании. М. : Физматгиз, 1960. 490 с.

8. Чивилихин С. А., Попов И. Ю., Гусаров В. В. Динамика скручивающихся нанотрубок в вязкой 
жидкости // Докл. АН. 2007. Т. 412, № 2. С. 201203.

9. Попов Ю. И., Розыгина О. А., Чивилихин С. А., Гусаров В. В. Солитоны в стенке нанотрубки и стоксово течение в ней // Письма в ЖТФ. 2010. Т. 36, вып. 18. С. 42-54.

10. Блинков Ю. А., Мозжилкин В. В. Генерация разностных схем для уравнения Бюргерса построением базисов Грёбнера // Программирование. 2006. T. 32, № 2. C. 71-74

11. Gerdt V. P., Blinkov Yu. A., Mozzhilkin V. V. Gröbner Bases and Generation of Difference Schemes for Partial Differential Equations // Symmet- ry, Integrability and Geometry : Methods and Applications. 2006. Vol. 2. 26 p. URL: http:// www.emis.de / journals / SIGMA / 2006 / Paper051/ index.html (дата обращения: 03.03.2015).

12. Gerdt V. P., Blinkov Yu. A. Gröbner Bases and Involution and difference schemes for the NavierStokes equations // Computer Algebra in Scientific Computing. Lecture Notes in Computer Science. 2009. Vol. 5743. P. 94-105.

13. Gerdt V. P., Robertz D. A Maple Package for Computing Gröbner Bases for Linear Recurrence Relations // Nuclear Instruments and Methods in Physics Research. 2006. Vol. A559. P. 215-219.

\title{
Nonlinear Deformation Waves in a Geometrically and Physically Nonlinear Viscoelastic Cylindrical Shell Containing Viscous Incompressible Fluid and Surrounded by an Elastic Medium
}

\section{A. Yu. Blinkova ${ }^{1}$, Yu. A. Blinkov² , S. V. Ivanov² , L. I. Mogilevich ${ }^{3}$}

\begin{abstract}
${ }^{1}$ Saratov State Technical University named after Gagarin Yu. A., 77, Politekhnicheskaya st., 410054, Saratov, Russia, anblinkova@yandex.ru

${ }^{2}$ Saratov State University, 83, Astrakhanskaya st., 410012, Saratov, Russia, BlinkovUA@info.sgu.ru, evilgraywolf@gmail.com

${ }^{3}$ Volga Branch of Moscow State University of Railway Communications, 1a, Astrakhanskaya st., 410004, Saratov, Russia, Mogilevich@sgu.ru
\end{abstract}

\begin{abstract}
The present study is devoted to analysis of nonlinear deformation of longitudinal waves in a cylindrical shell surrounded by an elastic medium and containing viscous incompressible fluid inside. The physical properties of the shell are defined by the equations of quadratic theory of viscoelasticity, which takes into account the linear elastic volume strain. The problem of wave propagation in viscoelastic and nonlinear thin-walled structures, including cylindrical shells, without interaction with the viscous incompressible fluid are considered from the perspective of earlier theory of solitons. The presence of fluid requires the development of new mathematical models and computer simulation of the processes occurring in the system.
\end{abstract}

Key words: nonlinear waves, viscous incompressible fluid, viscoelastic cylindrical shell surrounding elastic medium.

This work was supported by the Russian Foundation for Basic Research (projects no. 13-01-00049-a).

\section{References}

1. Zemlyanukhin A. I., Mogilevich L. I. Nelineinye volny $v$ tsilindricheskikh obolochkakh: solitony, simmetrii, evoliutsiia [Nonlinear Waves in Cylindrical Shells : Solitons, Symmetry, Evolution]. Saratov, Saratov State Tech. Univ. Press, 1999, 132 p. (in Russian).

2. Arshinov G. A., Zemlyanukhin A. I., Mogilevich L. I. Two-dimensional Solitary Waves in Nonlinear Viscoelastic Deformable Environment. Akusticheskii zhurnal [Acoustical Physics], 2000, vol. 46, no. 1, pp. 116-117 (in Russian).

3. Arshinov G. A., Mogilevich L. I. Staticheskie $i$ dinamicheskie zadachi viazkouprugosti [Static and Dynamic Problems of Viscoelasticity]. Saratov, Saratov State RGM Univ. Press, 2000, 152 p. (in Russian).

4. Loitsyansky L. G. Mekhanika zhidkosti i gaza [Fluid Mechanics]. Moscow, Drofa, 2003, 840 p. (in Russian). DOI: 10.7463/0115.0755210.
5. Volmir A. S. Nelineinaia dinamika plastinok $i$ obolochek [Dynamics of Plates and Shells]. Moscow, Nauka, 1972, 432 p. (in Russian).

6. Moskvitin V. V. Soprotivlenie viazko-uprugikh materialov [Resistance Vyzko-elastic materials]. Moscow, Nauka, 1972, 328 p. (in Russian).

7. Vlasov V. Z., Leont'ev N. N. Balki, plity $i$ obolochki na uprugom osnovanii [Beams, Plates and Shells on Elastic Foundation]. Moscow, Fizmatgiz, 1960, 490 p. (in Russian).

8. Chivilikhin S. A., Popov I. Yu., Gusarov V. V. Dynamics of nanotube twisting in a viscous fluid. Doklady Physics, 2007, vol. 52, no. 1, pp. 60-62.

9. Popov I. Y., Rodygina O. A., Chivilikhin S. A., Gusarov V. V. Soliton in a nanotube wall and stokes flow in the nanotube. Technical Physics Letters, 2010, vol. 36, no. 9, pp. 852-855. DOI: 10.1134/S1063785010090221. 
10. Blinkov Yu. A., Mozzhilkin V. V. Generation of Difference Schemes for the Burgers Equation by Constructing Gröbner Bases. Programming and Computer Software, 2006, vol. 32, no. 2, pp. 114117. DOI: $10.1134 /$ S0361768806020095.

11. Gerdt V. P., Blinkov Yu. A., Mozzhilkin V. V. Gröbner Bases and Generation of Difference Schemes for Partial Differential Equations. Symmetry, Integrability and Geometry: Methods and Applications, 2006, vol. 2, 26 p. URL: http://www.emis.de/journals/SIGMA/2006
/Paper051/index.html (Accessed 02, March, 2015). DOI: 10.3842/SIGMA.2006.051.

12. Gerdt V. P., Blinkov Yu. A. Involution and Difference Schemes for the Navier-Stokes Equations. Computer Algebra in Scientific Computing, Lecture Notes in Computer Science, 2009, vol. 5743, pp. 94-105. DOI: 10.1007/978-3-642-04103-7_10.

13. Gerdt V. P., Robertz D. A Maple Package for Computing Gröbner Bases for Linear Recurrence Relations. Nuclear Instruments and Methods in Physics Research, 2006, vol. A559, pp. 215-219. DOI: 10.1016/j.nima.2005.11.171.

УДК 539.374

\title{
ИМПУЛЬСНЫЕ ВОЗДЕЙСТВИЯ НА ТРЕХСЛОЙНЫЕ КРУГОВЫЕ ЦИЛИНДРИЧЕСКИЕ ОБОЛОЧКИ В УПРУГОЙ СРЕДЕ
}

\section{Д. В. Леоненко ${ }^{1}$, Э. И. Старовойтов ${ }^{2}$}

\begin{abstract}
${ }^{1}$ Доктор фризико-математических наук, просрессор кафредры строительной механики Белорусского государственного университета транспорта, Гомель, leoden@tut.by

${ }^{2}$ Доктор фризико-математических наук, заведующий кафредрой строительной механики Белорусского государственного университета транспорта, Гомель, edstar@mail.by

Рассмотрены вынужденные колебания трехслойной цилиндрической оболочки в упругой безынерционной среде Винклера, возникающие под действием импульсных нагрузок. Для изотропных несущих слоев приняты гипотезы Кирхгофа-Лява. В толстом заполнителе учитываются работа поперечного сдвига и обжатие по толщине. Изменение перемещений принято линейным по поперечной координате. На границах контакта используются условия непрерывности перемещений. Получен ряд аналитических решений и проведен их численный анализ.
\end{abstract}

Ключевые слова: колебания, трехслойная цилиндрическая оболочка, собственные частоты, импульсные нагрузки.

\section{ВВЕДЕНИЕ}

Трехслойные пластины и оболочки нашли широкое применение в современных отраслях промышленности. Это обусловливает необходимость разработки методов их расчета. Различные модели упругих трехслойных конструкций предложены в [1]. Монографии [2-4] посвящены исследованию статического и динамического деформирования трехслойных пластин и оболочек, не связанных с упругими средами. В монографии [5] рассмотрены различные модели упругой среды, с которой связаны элементы конструкций при деформировании, в том числе исследовано деформирование однородных конструкций на упругом основании. Задачи квазистатического деформирования трехслойных стержней и пластин на упругом основании разобраны в работах [6-9]. Статья [10] посвящена гармоническим колебаниям физически круглых нелинейных трехслойных пластин. Нестационарные колебания упругой среды, ограниченной двумя эксцентричными сферическими поверхностями, и распространение нестационарных волн в упругом слое рассмотрены в $[11,12]$.

Одной из самых важных с практической точки зрения задач динамики является исследование спектра частот собственных колебаний. Ее решение позволяет определить собственные частоты и формы, знание которых необходимо для решения задач о колебаниях трехслойных конструкций при различных видах внешних воздействий. Здесь рассмотрены вынужденные колебания трехслойной цилиндрической оболочки в упругой среде при импульсных внешних нагрузках.

\section{1. ПОСТАНОВКА ЗАДАЧИ}

В тонких изотропных несущих слоях трехслойной круговой цилиндрической оболочки приняты гипотезы Кирхгофа - Лява. В толстом заполнителе учитываются работа поперечного сдвига и обжатие по толщине. Изменение перемещений принято линейным по поперечной координате. На границах контакта слоев используются условия непрерывности перемещений. Деформации малые. 